\title{
SOME ASPECTS OF THE FRENCH FLEXIBLE BAG WAVE-ENERGY DEVICE*
}

\author{
$\mathrm{BY}$ \\ D. C. SHAW \\ Imperial College, London, England
}

\begin{abstract}
French (1977) has suggested a channel form wave energy absorber in which power is removed from the wave front by flexible bags along the channel walls. He has given a simple theoretical model for such a device which has, however, several drawbacks which we attempt to remedy. Several two-dimensional models of the channel are examined in which one or more of the dimensions are many wavelengths long. In particular, it is possible to apply realistic boundary conditions at the channel walls and obtain relationships between the wall stiffness $\mu$ and the decay rate of the wave front, $\sigma$. Two main methods are used; the variational method developed by Evans and Morris (1972) and the Wiener-Hopf method, as modified by Jones (1952).
\end{abstract}

1. Introduction. A special type of wave-energy absorber, suggested by M. J. French, consists of a rigid channel whose walls are lined with flexible bags. The channel faces into the waves, and as the surface of the water rises and falls, so the bags expand and contract, driving gas turbines. In this way, energy is gradually removed from the wave front as it passes down the channel. From the structural point of view, such a device is very attractive since the channels are aligned parallel to the incoming waves so there are no complicated mooring requirements. It may even be possible for it to align itself automatically. Mathematically, the problem is fully three-dimensional, however, so precise theoretical work is difficult.

Probably the simplest theoretical model of a wave energy device is given by Salter (1974), where an absorbing surface moves in a vertical plane parallel to the waves and is shaped neither to reflect nor transmit them. A more complicated system has also been considered by French (1977) in which the vertical plane containing the motion is perpendicular to the incoming wave direction. The confining surfaces are in pairs defining channels along which boundary conditions are imposed which attenuate the wave as it travels along them.

\footnotetext{
* Received May 14, 1984.
} 
The simple model problem which French (1977) considers is illustrated in Fig. 1, and is defined by the following boundary conditions. On the walls of the channel, which are defined as the planes $y= \pm b$, the normal velocity of the fluid is prescribed, corresponding to a given assumed motion of the wall bags. The $z$-axis is vertically upwards while the $x$-axis is parallel to the planes and the direction of propagation of the waves. The time dependence of the waves is assumed to be

$$
\Phi(\mathbf{r}, t)=\operatorname{Re}\{\phi(\mathbf{r}) \exp (i k(x-c t))\}
$$

and henceforth we suppress the $e^{-i k c t}$ factor. Since the amplitude of the waves is small, the surface boundary condition is

$$
\frac{\partial \phi}{\partial z}-n \phi=0 \quad(x=0)
$$

where $n=k^{2} c^{2} / g$, and since the planes are assumed to be infinitely deep, the potential can be set as $\phi(x, y, z)=e^{n z} \phi(x, y)$, which satisfies (1.2) automatically. Now French (1977) assumes that, in some way, the walls absorb energy so that the wave amplitude decays like $e^{-s x}$. More specifically, the boundary conditions at the walls $y= \pm b$ are set as:

$$
\Phi_{y}= \pm A \operatorname{Re}(1+i) \alpha \sinh (1+i) \alpha b \exp [-s x+n z+i k(x-c t)]
$$

where

$$
k^{2}=s^{2}+n^{2}, \quad k^{2}=g n / c^{2}, \quad \alpha=(k s)^{1 / 2} .
$$

The reason for assuming these apparently unlikely boundary conditions is that the potential is easily found to be

$$
\begin{array}{ll}
\phi=A \cosh (1+i) \alpha y E \quad(-b<y<b), & \\
\phi=-\frac{1}{2} A\left(1-e^{2(1+i) \alpha b}\right) \exp [-(1+i) \alpha y] E & (y>b), \\
\phi=-\frac{1}{2} A\left(1-e^{2(1+i) \alpha b}\right) \exp [(1+i) \alpha y] E & (y<-b),
\end{array}
$$

where

$$
E=\exp [-s x+n z+i k x] .
$$

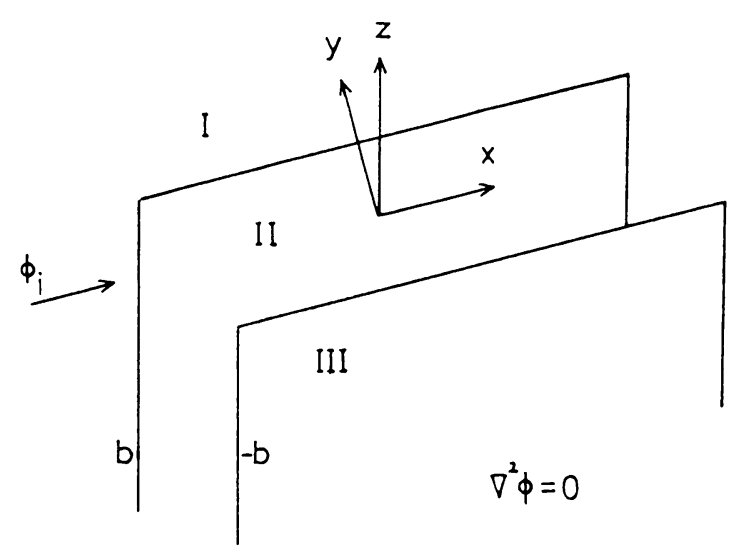

FIG. 1. French's (1977) model 
The constant $A$ can therefore be regarded as the amplitude of the wave at the origin. In the region marked III on Fig. 1, where $-b<y<b$, the potential satisfies (1.3), whereas in the regions II $(y<-b)$ and $I(y>b), \phi$ decays as $|y| \rightarrow \infty$. French (1977) then uses the results (1.5) to calculate predicted values for power absorption and efficiency, which give results in quite close agreement with experiments.

Theoretically, the model has two major drawbacks. First, the exponential damping in $x$, $e^{-s x}$, leads to infinite potentials at the end $x=-\infty$. It would be more satisfactory if some account could be taken of the open end of the channel facing the waves, rather than ignoring it and letting $\phi \rightarrow \infty$ there. Also, the channel walls considered here are infinitely deep, whereas in practice they are relatively shallow, about $2-10 \%$ of a wavelength deep. In applications, the spacing between the walls is sometimes small (i.e. $\alpha b \ll 1$ ) for single channels, or quite wide ( $\alpha b \gg 1$ ) where a pair or channels is used, or, say, a wall of the tank in the laboratory needs consideration.

Another problem is that the boundary conditions (1.3) assume that the potential decays expnentially as $e^{n z}$ with depth, and that the $x$-dependence is exponential. The actual boundary condition at a wall depends in fact on the equations of motion of the flexible bags used to convert power. Suppose for example that the bags obey a mass-acceleration law like

$$
m \ddot{\zeta}=K \zeta+D \dot{\zeta}
$$

where $\zeta$ is the displacement of the wall. Then the force is proportional to the variable part of the pressure, $\rho^{\partial \phi} / \partial t$, and the displacement is proportional to the potential. Since $\dot{\zeta}$ is just $\partial \phi / \partial y$ at the wall, (1.6) becomes, in terms of $\phi$,

$$
\phi_{y}+\mu \phi=0 \quad(y= \pm b)
$$

where $\mu$ is a complex number related to $m, \rho, K$ and $D$. It is interesting to see, therefore, whether an impedance type boundary condition like (1.7) does in fact lead to exponential damping in $x$. In what follows, we consider both boundary conditions like (1.3), called type (a) (for comparison with French's (1977) results) and the boundary conditions (1.7), called type (b).

In this paper, some two-dimensional versions of the channel are considered. First, an infinitely long but not infinitely deep channel is investigated. Then the $x$-dependence can be separated out of the solutions, leaving a two-dimensional problem in the $(y, z)$-plane. The case $\alpha b \gg 1$ of wide spacing can be dealt with using a variational principle similar to that used by Evans and Morris (1972a, b). When the spacing is narrow, a matching method can be used between an inner region near the bottom of the channel and an outer region where the channel appears as a single strip.

We can also deal with a channel which is infinitely deep, but only semi-infinitely long. In this case, the $z$-dependence can be separated out, leaving a problem equivalent to the diffraction of sound by a semi-infinite waveguide. This can be solved using a standard Wiener-Hopf technique both with type (a) and type (b) boundary conditions. Finally, using a method due to Jones (1952), the semi-infinite solution can be extended to the case where the channel is many wavelengths long, but finite. 
2. Infinitely long, finitely deep channels of type (a).

(i) Widely-spaced walls. In this case, the $x$-dependence can be separated out:

$$
\phi(x, y, z)=\phi(y, z) \exp [-s x+i k x] .
$$

A diagram showing a $(y, z)$ cross-section is given in Fig. 2. $(y, z)$ has been replaced in this section by $(x,-y)$, so that the $y$-axis is vertically downwards. On the sides of the channel, which has depth 1 , the boundary conditions (1.3) are

$$
\begin{aligned}
\phi_{x} & = \pm(A \operatorname{Re}(1+i) \alpha \sinh (1+i) \alpha b) e^{-n y} \\
& = \pm B e^{-n y} \quad(x= \pm b, 0<y<l)
\end{aligned}
$$

where the factor $\exp \{-s x+i k x\}$ has been left out, according to (2.1). This modified potential satisfies the partial differential equation

$$
\begin{aligned}
\nabla^{2} \phi(x, y, z) & =\left\{\frac{\partial^{2}}{\partial y^{2}}+\frac{\partial^{2}}{\partial z^{2}}+(s-i k)^{2}\right\} \phi(y, z) \\
& =\left\{\nabla_{1}^{2}-\nu^{2}\right\} \phi=0
\end{aligned}
$$

where $\nu=k+i$, and $\nabla_{1}^{2}$ is a two-dimensional $\mathrm{L}$ aplacian operator.

Since the problem is linear, we can furthermore set $B=1$ and then at the end multiply our result by any value of $B$. Far away from the channel, its influence must decay and so

$$
\phi \sim A_{ \pm} e^{-n y(1 \mp i) \alpha x} \quad(x \rightarrow \pm \infty)
$$

where $\alpha=(k s)^{1 / 2}$ as in (1.4) and $A_{+}=A_{-}$, in fact, by symmetry. Finally, on the free surface,

$$
\frac{\partial \phi}{\partial y}+n \phi=0 \quad(y=0) .
$$

The objective of this calculation is to evaluate the power absorbed by each channel wall. The force on each wall, per unit area, is proportional to $\phi$, and the local velocity is $\phi_{x}$ as in (2.2). The compression force on the channel sides is thus proportional to $\phi(b+0, y)-$ $\phi(b-0, y)$, and the power per unit length to

$$
P=\operatorname{Re} \int_{0}^{l} e^{-n y}(\phi(b+0, y)-\phi(b-0, y)) d y .
$$

When the spacing $2 b$ is large, it is best to formulate the problem as an integral equation.

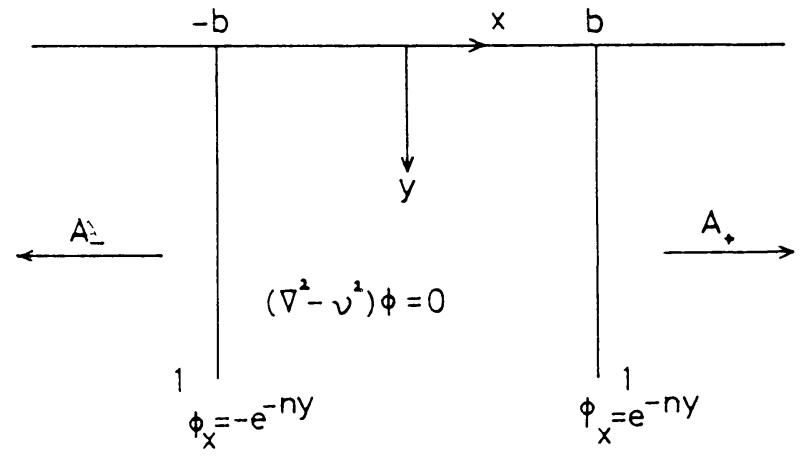

FIG. 2. Finitely deep, infinitely long channel 
Using a method similar to that used by Evans and Morris (1972a), we can derive two equations:

$$
\begin{aligned}
0= & -\frac{2}{\pi} \int_{0}^{\infty}\left\{\int_{l}^{\infty}\left(F(t)-e^{-n t}\right)(s \cos s t-n \sin s t) d t\right\} \\
& \times \frac{(s \cos s y-n \sin s y)\left(1+\operatorname{coth} s_{1} b\right)}{s_{1}\left(s^{2}+n^{2}\right)} d s \\
& +A(1+\operatorname{coth}(1+i) \alpha b) e^{-n y} \\
0= & \frac{2}{\pi} \int_{0}^{\infty}\left\{\int_{0}^{l} G(t)(s \cos s t-n \sin s t) d t\right\} \\
& \times \frac{s \cos s y-n \sin s y}{\left(1+\operatorname{coth} s_{1} b\right)\left(s^{2}+n^{2}\right)} d s \\
+ & ((1+i) \alpha A+1) e^{-n y}
\end{aligned}
$$

Equations (2.7) and (2.8) are equations of the first kind for $F$ and $G$, and either one, if it could be solved exactly, would give a solution to the problem. Unfortunately, we are in practice limited to approximate solutions only, and so we use a method due to Evans and Morris (1972a, b). First re-scale $F$ and $G$ so that $f$ and $g$ are given by

$$
\begin{aligned}
& f(t)-e^{-n t}=\frac{2}{\pi} A^{-1}\{1+\operatorname{coth}(1+i) \alpha b\}^{-1}\left(F(t)-e^{-n t}\right), \\
& g(t)=-\frac{2}{\pi}\{(1+i) \alpha A+1\}^{-1} G(t) .
\end{aligned}
$$

Then if $h(t)=e^{-n t}$, Eqs. (2.7) and (2.8) become, symbolically,

$$
L(f-h)=h, \quad M g=h
$$

where $L$ and $M$ are the integral operators. Defining also the inner product $\langle f, g\rangle_{1}=$ $\int_{0}^{l} f \bar{g} d y$ and $\langle f, g\rangle_{2}=\int_{l}^{\infty} f \bar{g} d y$, we obtain

$$
\langle f-h, h\rangle_{2}=\frac{1}{\pi n} C, \quad\langle g, h\rangle_{1}=\frac{1}{\pi n C}
$$

where

$$
C=\frac{1+(1+i) \alpha A}{A(1+\operatorname{coth}(1+i) \alpha b)} .
$$

Bear in mind that the quantity we wish to estimate is $\operatorname{Re}\langle g, h\rangle_{1}=\operatorname{Re} C^{-1} / \pi n$. We can now use the Schwinger variational principle, which states that if $\psi$ is an approximate solution to $L f=h$, then a better approximation to $\langle f, h\rangle$ is given by $\langle f, h\rangle$ $\sim\langle\psi, h\rangle^{2} /\langle\psi, L \psi\rangle$. Full details of the theory behind this result can be found in Jones (1964), but it needs to be slightly modified since here the functions are complex.

Notice that the only complex part of the operators $L$ and $M$ is in the factor $s_{1}=\left(s^{2}+\nu^{2}\right)^{1 / 2}$. Now $\bar{s}_{1}=\left(s^{2}+\bar{\nu}^{2}\right)^{1 / 2}$ but since $n^{2}=(\operatorname{Re} \nu)^{2}-(\operatorname{Im} \nu)^{2}$, changing $\nu$ to 
$\bar{\nu}$ does not alter $n$. Therefore,

$$
L f=e^{-n y} \Rightarrow \bar{L} f=e^{-n y} .
$$

Writing $f_{r}=\operatorname{Re} f$ and $f_{i}=\operatorname{Im} f$, this means that

$$
L f_{r}=e^{-n y} \quad \text { and } \quad L f_{i}=0 .
$$

If the inner product is such that $\langle a, L b\rangle=\langle\bar{L} a, b\rangle$ for any $a$ and $b$, then $\operatorname{Im}\langle f, h\rangle$ $=\left\langle f_{i}, L f\right\rangle=\left\langle\bar{L} f_{i}, f\right\rangle=\langle 0, f\rangle=0$. The inner products \langle\rangle$_{2}$ and \langle\rangle$_{1}$ have this property with $L$ and $M$ respectively, and so $C$ in (2.11) is real.

Notice also that for any function $a(y)$,

$$
\langle a, L a\rangle_{2}=\int_{0}^{\infty}\left|\int_{1}^{\infty} a(y)(s \cos s y-n \sin s y) d y\right|^{2} \frac{\left(1+\operatorname{coth} s_{1} b\right)}{s_{1}\left(s^{2}+n^{2}\right)} d s
$$

so that

$$
\operatorname{Re}\langle a, L a\rangle_{2} \geqslant 0 \quad \forall a(y) .
$$

Suppose $f_{1}=a_{1} \psi$, where $a_{1}=\langle\psi, h\rangle /\langle\psi, L \psi\rangle$. Then, following Jones (1964),

$$
\begin{aligned}
\operatorname{Re}\left\langle f-f_{1}, h\right\rangle & =\operatorname{Re}\left\{\langle f, L f\rangle-2\left\langle f_{1}, L f\right\rangle+\left\langle f_{1}, L f\right\rangle\right\} \\
& =\operatorname{Re}\left\langle f-f_{1}, L\left(f-f_{1}\right)\right\rangle \geqslant 0 .
\end{aligned}
$$

Therefore, the approximation $\left\langle f_{1}, h\right\rangle$ always has a real part smaller then $\langle f, h\rangle$; that is,

$$
\operatorname{Re} \frac{\langle\psi, h\rangle^{2}}{\langle\psi, L \psi\rangle} \leqslant\langle f, h\rangle
$$

An exact solution to this problem is available in the limit $\nu \rightarrow 0, b \rightarrow \infty$ where the barriers no longer feel the effect of each other and the fluid satisfies Laplace's equation. The solution to this single-barrier radiation problem is given by Ursell (1947) and is

$$
\begin{aligned}
\left(\frac{\partial \phi}{\partial x}\right)_{x=h}-e^{-n t} & =\psi(t)=t\left(t^{2}-l^{2}\right)^{-1 / 2} \quad(l<t<\infty) \\
\phi(b+0, t)-\phi(b-0, t) & =\chi(t)=\left(l^{2}-t^{2}\right)^{1 / 2} \quad(0<t<l) .
\end{aligned}
$$

Using the method of Evans and Morris (1972a, b),

$$
\begin{aligned}
& \langle\psi, L \psi\rangle_{2}=\int_{0}^{\infty} \frac{t^{2} J_{1}^{2}(t)(1+\operatorname{coth} \lambda t)}{\left(t^{2}+\mu^{2}\right)\left(t^{2}+\nu^{2} l^{2}\right)^{1 / 2}} d t=D(\lambda, \mu, \nu l), \\
& \langle\chi, M \chi\rangle_{1}=\int_{0}^{\infty} \frac{J_{1}^{2}(t)\left(t^{2}+\nu^{2} l^{2}\right)^{1 / 2}}{(1+\operatorname{coth} \lambda t)\left(t^{2}+\mu^{2}\right)} d t=E(\lambda, \mu, \nu l)
\end{aligned}
$$

where $\mu=n l, \lambda=b / l$.

In (2.10), using the approximations $\psi$ and $\chi$ for $f-e^{-n t}$ and $g$ respectively, this gives

$$
\operatorname{Re} C_{2} \leqslant C \leqslant \operatorname{Re} C_{1}
$$

where

$$
C_{2}=K_{1}^{2}(\mu) / \pi^{2} D, \quad C_{1}=E / \pi^{2} I_{1}^{2}(\mu)
$$


This gives an exact solution when $\operatorname{Re} C_{1}=\operatorname{Re} C_{2}$, which only occurs in the limit $\lambda \rightarrow \infty$. The approximation can therefore be expected to be good if the barriers are far apart. Once $C$ is estimated in this way, $\langle g, h\rangle_{1}$, which is the power integral, can be found from (2.11) and so the approximation is complete.

(ii) Closely-spaced walls. When the walls are close together, they appear to be a single wall with a source-like singularity at the tip where the bottom of the channel is. We can therefore use a method due to Thomas (1981), who had a similar situation with a narrow fully submerged duct. A diagram showing the present problem and the three regions to be matched together is given in Fig. 3 .

Firstly, in region $I$ we re-scale the coordinates to give

$$
\varepsilon \xi=x, \quad \eta=y
$$

where $\varepsilon$ is the small spacing between the walls. Using this scaling, the walls are now separated to $\xi= \pm 1$, and the local potential satisfies

$$
\begin{aligned}
\phi_{1 \xi \xi}=0 & & (\eta>0) \\
\phi_{1 \eta}+n \phi_{1}=0 & & (\eta=0), \\
\phi_{1 \xi}=0 & & (\xi= \pm 1,0<\eta<l) .
\end{aligned}
$$

A solution to this problem, valid for $|\xi|<1,0<\eta<1$ but which breaks down near the bottom of the channel is

$$
\phi_{1}=C(n \eta-1) \quad(|\xi|<1,0<\eta<1, C \text { constant }) .
$$

This solution does not, of course, have any waves so it must be matched to the outside region via the tip region II. Here we scale $x$ and $y$ so that

$$
\varepsilon X=x, \quad \varepsilon Y=y-l .
$$

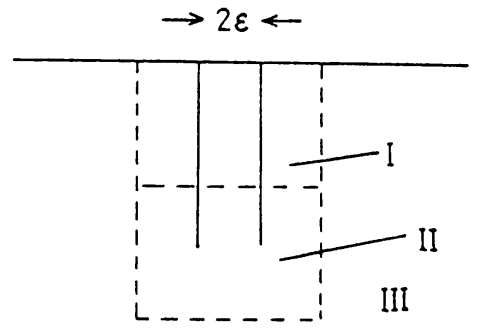

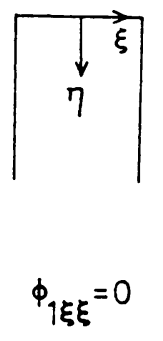

I

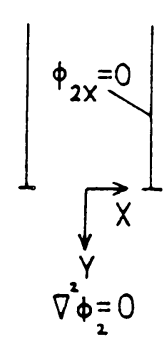

II

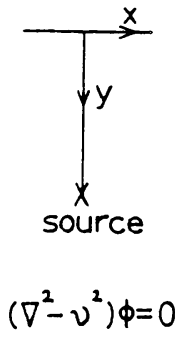

III

FIG. 3. Closely-spaced walls 
The problem now is of a semi-infinitely long channel with sides along $X= \pm 1, Y>0$. In region II, the local potential satisfies

$$
\begin{aligned}
& \left(\frac{\partial^{2}}{\partial X^{2}}+\frac{\partial^{2}}{\partial Y^{2}}\right) \phi_{2}=0, \\
& \phi_{2 X}=0 \quad(|X|=1, Y>0), \\
& \phi_{2} \sim C(n y-1) \quad(|X|<1, Y \rightarrow-\infty) .
\end{aligned}
$$

Solutions to problems of this sort are given in Lamb (1932) in implicit form, with far-field behaviours given by Newman (1974). If, as $Y \rightarrow-\infty$ within the tube,

$$
\phi_{2} \sim-\frac{\pi Y}{e_{1}}+\frac{1+e_{2}}{e_{1}} \quad(y \rightarrow-\infty,|X|<1)
$$

where $e_{1}$ and $e_{2}$ are arbitrary scaling constants, then the behaviour at large distances outside the tube is given by

$$
\phi_{2} \sim-\frac{1}{e_{1}} \log \pi R+\frac{e_{2}}{e_{1}} \quad\left(R=\left(X^{2}+Y^{2}\right)^{1 / 2} \rightarrow \infty\right)
$$

Since $C(n y-1)=C(n \varepsilon Y+n l-1)$, in definition $(2.25)$ we set

$$
1+e_{2}=\pi(1-n l) / n \varepsilon \text {. }
$$

The behaviour (2.26) suggests now that the outer problem in region III is that of a single barrier with a source at its tip. The outer potential thus satisfies

$$
\begin{aligned}
\left(\nabla^{2}-\nu^{2}\right) \phi & =q \delta(x) \delta(y-l) \quad(y>0), \\
\phi_{y}+n \phi & =0 \quad(y=0), \\
\phi_{x} & = \pm e^{-n y} \quad(x= \pm 0,0<y<l) .
\end{aligned}
$$

The source strength $q$ must be found later by matching. We can then divide $\phi$ into a singular part, and a complementary function part which has no singularities:

$$
\phi=q \Phi=\phi_{C F} .
$$

The singular part, $\Phi$, can be found by placing an image source above the surface and adding a correction for the free surface condition:

$$
\begin{aligned}
\Phi= & -\frac{i}{4}\left(H_{0}^{(2)}\left[-i \nu\left\{x^{2}+(y-l)^{2}\right\}^{1 / 2}\right]+H_{0}^{(2)}\left[-i \nu\left\{\left(x^{2}+l\right)^{2}\right\}^{1 / 2}\right]\right) \\
& +\int_{0}^{\infty} \frac{Z(s) \cos s x e^{-s_{1} y}}{n+s_{1}} d s
\end{aligned}
$$

where

$$
Z(s)=\frac{i n}{\pi} \int_{0}^{\infty} H_{0}^{(2)}\left[-i \nu\left\{x^{2}+l^{2}\right\}^{1 / 2}\right] \cos s x d x
$$

Now the function $\Phi$ as in (2.30) satisfies $\Phi_{x}=0$ on $x=0$, so to provide the necessary boundary condition on the barrier we add a complementary function:

$$
\begin{aligned}
\phi_{C F}= & q \Phi+A e^{-n y \pm(1+i) \alpha x} \\
& +\int_{0}^{\infty} \frac{a(s)(s \cos s y-n \sin s y) e^{ \pm s_{1} x}}{s_{1}\left(s^{2}+n^{2}\right)} d s \quad(x \lessgtr 0) .
\end{aligned}
$$


Now let

$$
G(y)=\phi_{x}(+0, y)= \begin{cases}e^{-n y} & (0<y<l), \\ 0 & (l<y<\infty)\end{cases}
$$

(since the problem is even in $x$ ) and then

$$
G(y)=-(1+i) \alpha A e^{-n y}-\int_{0}^{\infty} \frac{a(s)(s \cos s y-n \sin s y)}{s^{2}+n^{2}} d s .
$$

We can invert (2.33) by Havelock's (1929) theorem as before, to give $a(s)$ and $A$ :

$$
a(s)=-\frac{2}{\pi} e^{-n l} \sin l s, \quad A=-\frac{1}{(1+i) \alpha}\left(1-e^{-2 n l}\right) .
$$

Similarly

$$
\begin{aligned}
F(y)= & \phi( \pm 0, y)=q \Phi(0, y)+A e^{-n y} \\
& +\int_{0}^{\infty} \frac{a(s)(s \cos s y-n \sin s y)}{s_{1}\left(s^{2}+n^{2}\right)} d s .
\end{aligned}
$$

If we define the power integral as $P=2 \int_{0}^{l} e^{-n y} F(y) d y$, then

$$
\begin{aligned}
\frac{1}{2} P= & q \int_{0}^{l} e^{-n y} \Phi(0, y) d y-\frac{\left(1-e^{-2 n l}\right)^{2}}{2 n(1+i) \alpha} \\
& -\frac{2}{\pi} e^{-2 n l} \int_{0}^{\infty} \frac{\sin ^{2} s l}{s_{1}\left(s^{2}+n^{2}\right)} d s
\end{aligned}
$$

by using (2.34).

We now have a solution to the outer problem, but it remains to calculate $q$ by matching. As $z \rightarrow 0$

$$
H_{0}^{(2)}(z) \sim 1-\frac{2}{\pi} i\left\{\log \left(\frac{1}{2} z\right)+\gamma\right\}
$$

where $\gamma$ is Euler's constant. Therefore, from (2.30) and (2.31),

$$
\phi \sim q k_{1} \log \pi r+q k_{2}+k_{3} \quad(r \rightarrow 0)
$$

where $r^{2}=x^{2}+(y-l)^{2}$ and

$$
\begin{aligned}
& k_{1}=-\frac{1}{2 \pi} \\
& k_{2}=-\frac{i}{4}\left(H_{0}^{(2)}(-2 i \nu l)+1-\frac{2}{\pi} i\left\{\gamma+\log \left(-\frac{i \nu}{2 \pi}\right)\right\}\right)+\int_{0}^{\infty} \frac{Z(s) e^{-s_{1} l}}{n+s_{1}} d s \\
& k_{3}=\frac{e^{-n l}\left(1-e^{-2 n l}\right)}{(1+i) \alpha}-\frac{2}{\pi} e^{-n l} \int_{0}^{\infty} \frac{\sin s l(s \cos s l-n \sin s l)}{s_{1}\left(s^{2}+n^{2}\right)} d s
\end{aligned}
$$

Comparing this with the result (2.36), and replacing $r$ by $\varepsilon R$, we see that

$$
q=\frac{-k_{3}}{k_{1} e_{2}+k_{2}-\frac{1}{2 \pi} \log \varepsilon}
$$


where $e_{2}$ is as in (2.27). The solution is now complete, although many of the integrals will have to be evaluated numerically to find $k_{1}, k_{2}$ and $k_{3}$. It is worth noting, however, that many of the integrals reduce to

$$
\int_{-\infty}^{\infty} \frac{e^{i m s l}}{\left(s^{2}+\nu^{2}\right)^{1 / 2}(s \pm i n)} d s=M_{ \pm}(m, \nu, l, n) .
$$

For example, $k_{3}$ can be written entirely as a sum of integrals of this form. By deforming the contour of integration around the branch cut from $\pm i \nu$ to $i \infty$,

$$
M_{+}(m, \nu, l, n)=2 \pi i \frac{e^{l m n}}{(1+i) \alpha} H(-m)+\frac{2 i}{\nu} I\left(\nu l|m|, \frac{n}{\nu}\right),
$$

where

$$
I(\alpha, \gamma)=\int_{1}^{\infty} \frac{e^{-\alpha t}}{\left(t^{2}-1\right)^{1 / 2}(t+\gamma)} d t=-\pi e^{\alpha \gamma} \int_{\alpha}^{\infty} e^{-\gamma t} K_{0}(t) d t
$$

which can be found in tabulated form. Here $H(x)$ is the Heaviside function, the first term being the residue from the pole at $s=-i n$ which need only be included if $m<0$ and the contour deformed into the lower half-plane.

3. Infinitely long, finitely deep channels of type $(b)$.

(i) Widely-spaced walls. We repeat the analysis of Sec. 2(i) now, but replace the boundary condition on the walls by

$$
\phi_{x} \pm \mu \phi=0 \quad(x= \pm b, 0<y<l) .
$$

The problem is again even, but this time $\phi_{x}$ is no longer necessarily continuous across the walls. Therefore, re-define $F(y)$ as

$$
F(y)=\left(\phi_{x}+\mu \phi\right)_{x=b} \quad(y>0) .
$$

Using the same method as in Sec. 2 , since $F=0$ on the barrier and $G=0$ off it.

$$
\begin{aligned}
0= & \frac{2}{\pi} \int_{0}^{\infty}\left\{\int_{l}^{\infty} F(t)(s \cos s t-n \sin s t) d t\right\} \\
& \times \frac{Q(s)(s \cos s y-n \sin s y)}{\left(\mu-s_{1}\right)\left(s^{2}+n^{2}\right)} d s+A N e^{-n y} \quad(l<y<\infty)
\end{aligned}
$$

and

$$
\begin{aligned}
0= & \frac{2}{\pi} \int_{0}^{\infty}\left\{\int_{0}^{l} G(t)(s \cos s t-n \sin s t) d t\right\} \\
& \times \frac{\left(\mu-s_{1}\right)(s \cos s t-n \sin s t)}{s_{1} Q(s)\left(s^{2}+n^{2}\right)}+A(\mu-(1+i) \alpha) e^{-n y} \\
& \quad(l<y<\infty)
\end{aligned}
$$

where

$$
Q(s)=1-\left(\mu-s_{1}\right) \cosh s_{1} b /\left(s_{1} \sinh s_{1} b+\mu \cosh s_{1} b\right)
$$


and

$$
\begin{array}{r}
N(\mu, \alpha, b)=1-(\mu-(1+i) \alpha) \cosh (1+i) \alpha b /((1+i) \alpha \sinh (1+i) \alpha b \\
+\mu \cosh (1+i) \alpha b) .
\end{array}
$$

Now we re-scale $F$ and $G$ so that

$$
\begin{aligned}
& f(t)=-\frac{2}{\pi}(A N)^{-1} F(t) \\
& g(t)=-\frac{2}{\pi} A^{-1}(\mu-(1+i) \alpha)^{-1} G(t)
\end{aligned}
$$

and then

$$
L f=h, \quad M g=h,
$$

where $L$ and $M$ are now given by (3.3) and (2.4), and

$$
\langle f, h\rangle_{2}=\frac{1}{\pi n} C, \quad\langle g, h\rangle_{1}=\frac{1}{\pi n C}
$$

where

$$
C=\frac{\mu-(1+i) \alpha}{N(\mu, \alpha, b)}
$$

Notice that in this problem, $\langle g, h\rangle_{1}$, which is related to the power integral, is known exactly in (3.7). $A$ is, of course, not determined since the problem is linear and homogeneous so $A$ is an arbitrary scale factor. It is more interesting, though, to regard this as an eigenvalue problem relating the damping rate $\operatorname{Im} \nu$ (or, equivalently, $\alpha$ ) with the stiffness of the walls. To get approximate solutions to this, we can use (3.7) in a variational principle, but it is not really clear what the best initial approximations to take are. For small $\mu$ and large spacings, a possibility is to use the same $\psi$ and $\chi$ as in (2.17). Then

$$
\begin{aligned}
& \langle\psi, L \psi\rangle_{2}=\int_{0}^{\infty} \frac{t^{2} J_{1}^{2}(t) Q(b t / l)}{\left\{l \mu-\left(t^{2}+\nu^{2} l^{2}\right)^{1 / 2}\right\}\left(t^{2}+n^{2} l^{2}\right)} d t=D, \\
& \langle\chi, M \chi\rangle_{1}=\int_{0}^{\infty} \frac{J_{1}^{2}(t)\left\{l \mu-\left(t^{2}+\nu^{2} l^{2}\right)^{1 / 2}\right\}}{Q(b t / l)\left(t^{2}+n^{2} l^{2}\right)} d t=E .
\end{aligned}
$$

For small $\mu$ and $\operatorname{Im} \nu$, only, it can then be shown that

$$
\begin{aligned}
& \operatorname{Re} C_{2} \leqslant \operatorname{Re} C \leqslant \operatorname{Re} C_{1}, \\
& \operatorname{Im} C_{1} \leqslant \operatorname{Im} C \leqslant \operatorname{Im} C_{2}
\end{aligned}
$$

where

$$
C_{2}=K_{1}^{2}(n l) / \pi^{2} D, \quad C_{1}=E / \pi^{2} I_{1}^{2}(n l) .
$$

Once $C$ is known, then from (3.7) $\alpha$ is known in a complicated implicit way. For small $\mu$, the integrals $D$ and $E$ are approximately those in (2.18), so a simpler and possibly quite accurate approximation may be found using (2.18) instead of (3.8). 
(ii) Closely-spaced walls. The only difference here from Sec. 2(ii) is in the outer problem, which now has the specification

$$
\begin{array}{rlrl}
\left(\nabla^{2}-\nu^{2}\right) \phi & =q \delta(x) \delta(y-l) \\
\phi_{y}+n \phi & =0 & (y=0), \\
\phi_{x} \pm \mu \phi & =0 & (x= \pm 0,0<y<l) .
\end{array}
$$

The singular part, $\Phi$, remains the same, but on $x=0$ :

$$
\left(\frac{\partial}{\partial x} \pm \mu\right) \phi_{C F}=\mp \mu q \Phi(0, y) \quad(x= \pm 0,0<y<l) .
$$

Letting $H(y)=\phi_{x}+\left.\mu \phi\right|_{x=0}$, as before, by Havelock's (1929) theorem

$$
\begin{aligned}
a(s) & =\frac{2}{\pi} \frac{s_{1}}{\mu-s_{1}} \int_{l}^{\infty}\{H(y)-\mu q \Phi(0, y)\}(s \cos s y-n \sin s y) d y \\
A & =\frac{2 n}{\mu-(1+i) \alpha} \int_{l}^{\infty}\{H(y)-\mu q \Phi(0, y)\} e^{-n y} d y .
\end{aligned}
$$

Also, if $G(y)=\phi_{x}(0, y)$,

$$
\begin{aligned}
a(s) & =-\frac{2}{\pi} \int_{0}^{l} G(y)(s \cos s y-n \sin s y) d y, \\
A & =\frac{-2 n}{(1+i) \alpha} \int_{0}^{l} G(y) e^{-n y} d y .
\end{aligned}
$$

The boundary conditions (3.10) imply that $F=0$ on the barrier, so that, again using Evans and Morris' (1972) results,

$$
\begin{aligned}
0= & A(\mu-(1+i) \alpha) e^{-n y}-\frac{2}{\pi} \int_{0}^{\infty}\left\{\int_{0}^{l} G(t)(s \cos s t-n \sin s t) d t\right\} \\
& \times \frac{\left(\mu-s_{1}\right)(s \cos s y-n \sin s y) d s}{s_{1}\left(s^{2}+n^{2}\right)} .
\end{aligned}
$$

Also, the problem is even in $x$, so below the barrier $G=0$, and using (3.12) this gives

$$
\begin{array}{r}
0=-(1-i) \alpha A e^{-n y}-\frac{2}{\pi} \int_{0}^{\infty}\left\{\int_{l}^{\infty}[H(t)-\mu q \Phi(0, t)](s \cos s t-n \sin s t) d t\right\} \\
\cdot \frac{s_{1}(s \cos s y-n \sin s y)}{\left(\mu-s_{1}\right)\left(s^{2}+n^{2}\right)} d s .
\end{array}
$$

No exact solution to this problem is possible as it was with type (a) boundary condition, so we seek a variational solution as above. Again, re-scale $G$ and $H$ so that

$$
\begin{aligned}
& g(t)=-\frac{2}{\pi} A^{-1}(\mu-(1+i) \alpha)^{-1} G(t), \\
& f(t)=\frac{2}{\pi}[(1+i) \alpha A]^{-1}(H(t)-\mu q \Phi(0, t)) .
\end{aligned}
$$


In the limits $\nu l \rightarrow 0, \mu l \rightarrow 0$ we could use $\psi$ and $\chi$ as in (2.30) as approximations. Results (3.8)-(3.9) now hold, except that

$$
\begin{aligned}
& C=-\frac{\mu-(1+i) \alpha}{(1+i) \alpha} \\
& D=\int_{0}^{\infty} \frac{t^{2} J_{1}^{2}(t)\left(t^{2}+\nu^{2} l^{2}\right)^{1 / 2}}{\left\{l \mu-\left(t^{2}+\nu^{2} l^{2}\right)^{1 / 2}\right\}\left(t^{2}+n^{2} l^{2}\right)} d t \\
& E=\int_{0}^{\infty} \frac{J_{1}^{2}(t)\left\{l \mu-\left(t^{2}+\nu^{2} l^{2}\right)^{1 / 2}\right\}}{\left(t^{2}+\nu^{2} l^{2}\right)^{1 / 2}\left(t^{2}+n^{2} l^{2}\right)} d t
\end{aligned}
$$

This does not help in finding $q$, however. If $\psi$ and $\chi$ are used as initial approximations, they give a value of $k_{3}=0$, which means $q=0$ and leads to a trivial inner solution.

Possibly a better idea is to use (2.32) as a first approximation to $G$. This gives a new value for $E$, namely

$$
E_{1}=\int_{0}^{\infty} \frac{e^{-2 n l} \sin ^{2} l t\left\{l \mu-\left(t^{2}+\nu^{2} l^{2}\right)^{1 / 2}\right\}}{\left(t^{2}+\nu^{2} l^{2}\right)\left(t^{2}+n^{2} l^{2}\right)} d t
$$

and the first approximation to $q$ remains as in (2.37). It is hard, though, to find a corresponding value for $D_{1}$ and so a lower bound for $C$ is not easy to calculate. There is no rigorous way of telling which approximation is better; that involving $(3.18 \mathrm{~b}-\mathrm{c})$ or (3.19). Possibly (3.19), which gives a non-zero value of $q$, is better for calculating the power, while for calculating $\alpha$ as a function of $\mu$, where $q$ is not so important, (3.18) may be better.

\section{Semi-infinitely long, infinitely deep channels.}

(i) Type (a) boundary conditions. If the channel is infinitely deep, then the $z$-dependence can be separated out:

$$
\phi(x, y, z)=e^{-k z} \phi(x, y)
$$

where here the $z$-axis points vertically downwards. (4.1) then automatically satisfies the free-surface condition, and also the two-dimensional partial differential equation

$$
0=\left\{\frac{\partial^{2}}{\partial x^{2}}+\frac{\partial^{2}}{\partial y^{2}}+k^{2}\right\} \phi(x, y)=\left(\nabla^{2}+k^{2}\right) \phi
$$

applies. A plan view of the channel is given in Fig. 4. The symmetry in $y$ implies that only half the plane needs to be considered. A wave $\phi_{i}=e^{i k x}$ is incident from the left, parallel

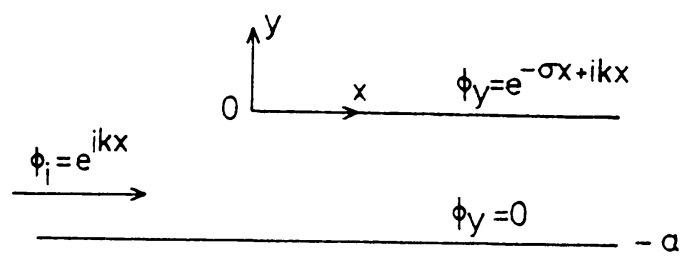

FIG. 4. Semi-infinitely long channel 
to the walls of the channel, and we write

$$
\phi=\phi_{i}+ \begin{cases}\phi_{1} & (-a<y<0), \\ \phi_{2} & (y>0),\end{cases}
$$

where $a$ is the half-width of the channel. The functions $\phi_{1}$ and $\phi_{2}$ then satisfy the boundary conditions

$$
\begin{gathered}
\phi_{1 y}=\phi_{2 y}=0(y=-a), \quad \phi_{2} \rightarrow 0 \quad(y \rightarrow \infty), \\
\phi_{2}=\phi_{1} \text { and } \phi_{2 y}=\phi_{1 y} \quad(y=0, x<0), \\
\phi_{1 y}=\phi_{2 y}=e^{-\sigma x+i k x} \quad(y=0, x>0) .
\end{gathered}
$$

Here (4.4c) is the type (a) wall condition and $\sigma$ is the damping constant. To improve convergence, let $k$ be slightly complex, $k=k_{r}+i \varepsilon$, and later let $\varepsilon \rightarrow 0$. The conditions at infinity are then

$$
\begin{aligned}
& \phi_{1} \text { and } \phi_{2} \sim V(x) e^{-\varepsilon x} \quad(x \rightarrow \infty)(V \text { algebraic }) \\
& \phi_{1} \text { and } \phi_{2}=O(1) \quad(x \rightarrow-\infty) .
\end{aligned}
$$

Finally, to fix the problem and ensure uniqueness, we require an edge condition at the lip of the channel at $(0,0)$. Energy considerations imply that

where $r^{2}=x^{2}+y^{2}$.

$$
|\nabla \phi|=O\left(r^{\alpha}\right) \quad(\alpha>-1)(r \rightarrow 0)
$$

Half-plane problems of this sort can be dealt with in a standard way by using the Wiener-Hopf method. Noble (1958) in fact gives a calculation for a similar problem in acoustics, but with a different boundary condition in place of (4.4c). Following Noble (1958) closely, we take the Fourier transform in $x$ of the problem and write

$$
\begin{aligned}
\Phi & =\int_{-\infty}^{\infty} \phi(x, y) e^{i s x} d x=\int_{-\infty}^{0} \phi e^{i s x} d x+\int_{0}^{\infty} \phi e^{i s x} d x \\
& =\Phi_{-}(s)+\Phi_{+}(s) .
\end{aligned}
$$

From the behaviour at infinity $(4.5 \mathrm{a}, \mathrm{b}), \Phi_{+}$is an analytic function of $s$ in the region $\operatorname{Re} s>0$, while $\Phi_{-}$is analytic for $\operatorname{Re} s<\varepsilon$. Notice that both $\Phi_{+}$and $\Phi_{-}$are analytic in the strip $0<\operatorname{Re}(s)<\varepsilon$. Using the usual method, we obtain a pair of coupled equations

$$
\begin{gathered}
\Phi_{1-}+\Phi_{1+}-\frac{\cosh \gamma a}{\gamma \sinh \gamma a} \Phi_{1-}^{\prime}=\frac{\cosh \gamma a}{\gamma \sinh \gamma a} \frac{i}{s+\nu}, \\
\Phi_{1-}+\Phi_{2-}+\frac{1}{\gamma} \Phi_{1-}^{\prime}=\frac{1}{\gamma} \frac{i}{s+\nu}
\end{gathered}
$$

where $\Phi_{1}$ and $\Phi_{2}$ are the transforms of $\phi_{1}$ and $\phi_{2}$ along $y=0$, and ' $=d / d y$. The square root in $\gamma=\left(s^{2}-k^{2}\right)^{1 / 2}$ is chosen so that $\gamma=-i k$ when $s=0$, with branch cuts along the lines $\pm k t(1<t<\infty)$. There is no known standard way of dealing with coupled equations like (4.8), but fortunately for channel problems a change of variable reduces (4.8) to a single equation. If we simply subtract (4.8b) from (4.8a), where $D=\Phi_{2+}-\Phi_{1+}$ is the transform of the potential difference across the channel wall. Now let

$$
K(s)=\frac{\sinh \gamma a}{\gamma a e^{\gamma a}}=K_{+}(s) K_{-}(s) .
$$


The factorisation of $K$ into $K_{+}$, which is analytic in $\operatorname{Re} s>0$, and $K_{-}$, analytic for $\operatorname{Res}<\varepsilon$, is given in the appendix. Rearranging (4.9) then gives

$$
\frac{\Phi_{1-}^{\prime}}{a(s-k) K_{-}(s)}+(s+k) K_{+}(s) D_{+}=-\frac{1}{a(s-k) K_{-}(s)} \frac{i}{s+\nu} .
$$

The sum decomposition of the right hand side can be done by inspection:

$$
\begin{aligned}
-\frac{1}{a(s-k) K_{-}(s)} \frac{i}{s+\nu}= & -\frac{i}{a(s+\nu)}\left\{\frac{1}{(s-k) K_{-}(s)}+\frac{M}{k+\nu}\right\} \\
& +\frac{i M}{a(k+\nu)(s+\nu)}
\end{aligned}
$$

where $M=1 / K_{-}(-\nu)$, and using this in (4.11) gives

$$
\begin{aligned}
& \frac{\Phi_{1-}^{\prime}}{a(s-k) K_{-}}+\frac{i}{a(s+\nu)}\left\{\frac{1}{(s-k) K_{-}(s)}+\frac{M}{k+\nu}\right\} \\
& =(s+k) K_{+} D_{+}+\frac{i M}{a(k+\nu)(s+\nu)} .
\end{aligned}
$$

The entire left hand side is analytic for $\operatorname{Re} s<\varepsilon$ and the right-hand side for $\operatorname{Re} s>0$, by definition. Since they are equal, this means that both sides are an entire function, $E(s)$, say.

In order to evaluate $E(s)$, we make use of the edge condition (4.6). This tells us that, for large $s$,

$$
D_{+}(s)=O(1 / s), \quad \Phi_{1-}^{\prime}=O\left(1 / s^{1+\alpha}\right) \quad(\alpha>-1) \quad(s \rightarrow \infty) .
$$

Also, $K_{ \pm}(s)=O\left(1 / s^{1 / 2}\right)(s \rightarrow \infty)$ so that both the right and left hand sides of (4.13) decay at infinity. Because $E$ is an entire function which tends to zero as $s \rightarrow \infty, E(s)=0$ by Liouville's theorem. In particular, the right-hand side of (4.13) implies that

$$
D_{+}(s)=\frac{i}{a(k+\nu) K_{-}(-\nu)} \frac{1}{K_{+}(s)(s+k)(s+\nu)}
$$

so that

$$
\begin{aligned}
\phi(x,+0)-\phi(x,-0) & =f(x) \\
& =\frac{i}{2 \pi a(k+\nu) K_{-}(-\nu)} \int_{-\infty+i \gamma}^{\infty+i \gamma} \frac{e^{-i s x}}{K_{+}(s)(s+k)(s+\nu)} d s .
\end{aligned}
$$

where $0<\gamma<\varepsilon$. It is fortunate that $f(x)$ is precisely the quantity of interest since it is proportional to the net compression force on the walls of the channel.

We can evaluate the integral in (4.16) by closing the contour in the lower half-plane. The integrand has a pole at $s=-\nu$, another at $s=-k$ and poles at the roots of $K_{+}(s)$ which lie at

$$
\alpha_{n}=\left(k^{2}-\frac{n^{2} \pi^{2}}{a^{2}}\right)^{1 / 2}, \quad n=1,2, \ldots
$$


A diagram of the contour around which the integral is taken is given in Fig. 5, where $\varepsilon \rightarrow 0$. Note that as $\varepsilon \rightarrow 0$, the pole at $s=-k$ gives a travelling wave component. If we are interested in the behaviour of $f(x)$ as $x \rightarrow \infty$, then the exact dimensions of the channel become important. If, for example,

$$
\pi^{2} / a^{2}-k^{2}>\sigma^{2}>0
$$

then the dominant pole, apart from the travelling wave, lies at $s=-\nu$;

$$
\begin{aligned}
& f(x) \sim \frac{1}{a(k+\nu)}\left\{\frac{e^{i \nu x}}{K_{-}(-\nu) K_{+}(-\nu)(k-\nu)}+\frac{e^{i k x}}{K_{-}(-\nu) K_{+}(-k)(-k+\nu)}\right\} \\
&=\frac{-a^{2}}{\left(k^{2}-\nu^{2}\right)^{1 / 2}}\left\{\frac{\exp i\left(k^{2}-\nu^{2}\right)^{1 / 2} a}{\sin \left(k^{2}-\nu^{2}\right)^{1 / 2} a} e^{i \nu x}-\frac{a^{-3}\left(k^{2}-\nu^{2}\right)^{-1 / 2}}{K_{+}(\nu) K_{-}(k)} e^{i k x}\right\} \\
&(x \rightarrow \infty) .
\end{aligned}
$$

If $\pi^{2} / a^{2}<k$, then the dominant pole comes from $\alpha_{1}$ and the behaviour is quite different. If we let $\sigma \rightarrow 0$ in (4.19), note that the expression tends to a finite value, as it should.

(ii) Type (b) boundary conditions. In this section, instead of the condition (4.4c) we use $\phi_{y}=\mu \phi$ on the channel walls, so that

$$
\phi_{1 y}=\mu \phi_{1}-\mu e^{i k x}, \quad \phi_{2 y}=\mu \phi_{2}-\mu e^{i k x} \quad(y=0, x>0) .
$$

As before, we obtain the pair of coupled equations:

$$
\begin{gathered}
\gamma \tanh \gamma a \Phi_{1-}-\Phi_{1-}^{\prime}-\frac{i \mu}{s+k}+(\gamma \tanh \gamma a-\mu) \Phi_{1+}=0, \\
\gamma \Phi_{1-}+\Phi_{1-}^{\prime}+\frac{i \mu}{s+k}+(\gamma+\mu) \Phi_{2+}=0 .
\end{gathered}
$$

At first sight, there seems to be no way of reducing these to a single equation, until they are re-arranged slightly

$$
\begin{array}{r}
(\gamma \tanh \gamma a-\mu) \Phi_{1-}-\left(\Phi_{1}^{\prime}-\mu \Phi_{1-}\right)-\frac{i \mu}{s+k}(\gamma \tanh \gamma a-\mu) \Phi_{1+}=0, \\
(\gamma+\mu) \Phi_{1-}+\left(\Phi_{1-}^{\prime}-\mu \Phi_{1-}\right)+\frac{i \mu}{s+k}+(\gamma+\mu) \Phi_{2+}=0,
\end{array}
$$

and now eliminating $\Phi_{1-}$ between these gives

$$
\left\{\frac{1}{\gamma+\mu}+\frac{1}{\gamma \tanh \gamma a-\mu}\right\}\left(F_{-}+\frac{i \mu}{s+k}\right)+G_{+}=0
$$

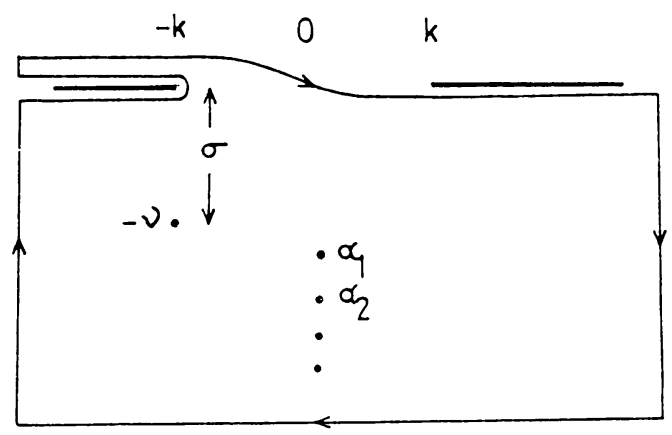

FIG. 5. Contour used for the integral (4.20) 
where

$$
F_{-}=\Phi_{1-}^{\prime}-\mu \Phi_{1-}, \quad G_{+}=\Phi_{2+}-\Phi_{1+} .
$$

It is really quite fortunate that this reduction can be carried out because, as mentioned earlier, there is no systematic method for dealing with coupled equations like (4.21). Re-write (4.23) as

$$
F_{-}+a\left(s^{2}-k^{2}\right) K(s) G_{+}+\frac{i \mu}{s+k}=0
$$

where

$$
K(s)=\frac{1}{\gamma a} e^{-\gamma a}\left(1+\mu \gamma^{-1}\right)\left(\sinh \gamma a-\mu \gamma^{-1} \cosh \gamma a\right) .
$$

$K$ can be factorised, as described in the appendix, into a product $K_{+}(s) K_{-}(s)$. Then (4.24) gives

$$
\frac{F_{-}}{a(s-k) K_{-}}+(s+k) G_{+} K_{+}+\frac{i \mu}{a\left(s^{2}-k^{2}\right) K_{-}}=0 .
$$

The necessary sum decomposition then gives

$$
\begin{aligned}
(s+k) & K_{+}(s) G_{+}(s)-\frac{i \mu}{2 k a K_{-}(-k)(s+k)} \\
& =\frac{i \mu}{2 k a(s+k)}\left\{\frac{1}{K_{-}(s)}-\frac{1}{K_{-}(-k)}\right\}-\frac{i \mu}{2 k a(s-k) K_{-}(s)}-\frac{F_{-}(s)}{a(s-k) K_{-}(s)} \\
& =E(s) .
\end{aligned}
$$

As before, $E(s)$ is an entire function and from the edge conditions and Liouville's theorem, $E=0$. The left hand side of (4.26) this time is

$$
G_{+}(s)=\frac{i \mu}{2 k a K_{-}(-k)(s+k)^{2} K_{+}(s)}
$$

so that the potential difference across the wall of the channel is

$$
f(x)=\frac{i \mu}{4 \pi k a K_{-}(-k)} \int_{-\infty+i \gamma}^{\infty+i \gamma} \frac{e^{-i s x}}{K_{+}(s)(s+k)^{2}} d s
$$

where $0<\gamma<\varepsilon$.

To evaluate the integral, we again make use of the contour in Fig. 5. Bear in mind that near $s=-k, K_{+}(s)=O\left((s+k)^{-3 / 2}\right)$ and the singularity there is integrable. Poles lie at the roots of $K_{+}(s)$, which are $\alpha_{0}=\left(k^{2}+\mu^{2}\right)^{1 / 2}$ and also the roots of

$$
a \beta_{n} \tanh a \beta_{n}=\mu a, \quad \alpha_{n}^{2}-k^{2}=\beta_{n}^{2} \quad(n=1,2, \ldots) .
$$

At least for $\mu$ small enough, $\alpha_{0}$ has a smaller imaginary part than any of the other poles. There is also a contribution from the branch cut integral, which is estimated for large $x$ by noting that near $s=-k$,

$$
K_{+}(s) \sim e^{\pi i / 4}(s+k)^{-3 / 2} \mu a^{-1 / 2} .
$$


We therefore get a travelling wave and a damped mode for large $x$ :

$$
f(x) \sim \frac{(k a)^{1 / 2}}{i \sqrt{\pi}} \frac{1}{K_{+}(k)} \frac{e^{i k x}}{(k x)^{1 / 2}}-\frac{\mu}{2 k a K_{-}(-k)} \frac{e^{-i \alpha_{0} x}}{K_{+}^{\prime}\left(\alpha_{0}\right)\left(\alpha_{0}+k\right)^{2}}(x \rightarrow \infty) .
$$

A significant feature of (4.19) and (4.29) is that a travelling wave is present rather than just an exponentially damped mode as predicted by French's fully infinite theory.

5. Finitely long, infinitely deep channels. If the channel is very long, but only finitely so, then we can use a method due to Jones (1952), who considered a similar diffraction problem by a finite waveguide. Noble (1958) re-formulated Jones's (1952) method in terms of Fourier transforms, and it is his formulation which we shall proceed to use here. The method was developed, however, for waves which strike the channel at a non-zero angle to the channel walls, so some modification is necessary for this case, where the waves are at grazing incidence.

Following as far as possible the notation of Noble (1958), consider a wave incident from the right, $\phi_{i}=e^{-i k x}$, on a channel extending from $x=+d$ to $x=-d$ and of width $2 a$. Define

$$
\begin{aligned}
& \Phi_{+}(s)=\int_{d}^{\infty} \phi(x, y) e^{i s(x-d)} d x, \\
& \Phi_{-}(s)=\int_{-\infty}^{-d} \phi(x, y) e^{i s(x+d)} d x, \\
& \Phi_{a}(s)=\int_{-d}^{d} \phi(x, y) e^{i s x} d x .
\end{aligned}
$$

$\Phi_{+}$is analytic for $\operatorname{Re} s>0, \Phi_{-}$for $\operatorname{Re} s<\varepsilon$ while $\Phi_{a}$ is analytic everywhere. Identical manipulations to those in section four lead to the equation

$$
\left\{\frac{1}{\gamma \tanh \gamma a-\mu}+\frac{1}{\gamma+\mu}\right\}\left[e^{i d s} \Psi_{+}+e^{-i d s} \Psi_{-}+F(s)\right]+D_{a}=0
$$

instead of (4.28), where

$$
\begin{aligned}
\Psi_{ \pm} & =\Phi_{1 \pm}^{\prime}-\mu \Phi_{1 \pm}, \\
D_{a} & =\Phi_{2 a}-\Phi_{1 a}, \\
F(s) & =\frac{i \mu}{s-k}\left(e^{i(s-k) d}-e^{-i(s-k) d}\right) .
\end{aligned}
$$

Re-arranging (5.2),

$$
e^{-i d s} \Psi_{-}+K(S) D_{a}+e^{i d s} \Psi_{+}+F(s)=0
$$

where

$$
K(s)=e^{-\gamma a}\left(1+\mu \gamma^{-1}\right)(\gamma \sinh \gamma a-\mu \cosh \gamma a) .
$$

The essence of Jones's (1952) method is the estimation of certain integrals, and to do this note that

$$
K_{-}(\zeta) \sim e^{i \pi / 4} \mu(\zeta-k)^{-1 / 2} \quad(\zeta \rightarrow k)
$$


Therefore

$$
\left\{K_{-}(\zeta)\right\}^{-1} \sim e^{-i \pi / 4} \mu^{-1}(\zeta-k)^{1 / 2}=h_{r}(\zeta-k)^{r+1 / 2}
$$

where

$$
r=0 \quad \text { and } \quad h_{r}=e^{-i \pi / 4} \mu^{-1} .
$$

There are three integrals to be estimated. The first two can be done just as in Noble (1958). However, care must be taken with the third integral because the pole of $F(s)$ in (5.3) coincides with the branch point at $s=k$ of $K_{-}(s)$ :

$$
\begin{aligned}
& \int_{i a-\infty}^{i a+\infty} \frac{e^{i \zeta l}}{(\zeta+\alpha)(\zeta+k) K_{-}(\zeta)} d \zeta \sim 2 \pi i R_{2}(\alpha), \\
& R_{2}(\alpha)=\frac{1}{2 \pi i} E_{r} W_{r-1}(-i(k+\alpha) l)
\end{aligned}
$$

and this value is different from that quoted in Noble (1958). Furthermore, the functions $P_{1}$ and $P_{2}$ become

$$
P_{1}(\alpha)=\frac{1}{\alpha-k}\left\{\frac{1}{K_{+}(\alpha)}-\frac{1}{K_{+}(k)}\right\}, \quad P_{2}(\alpha)=\frac{1}{(\alpha+k) K_{+}(\alpha)} .
$$

Then

$$
\begin{aligned}
-\Psi_{+}= & i \mu K_{+}(s)\left[e^{-k d} \frac{1}{s-k}\left\{\frac{1}{K_{+}(s)}-\frac{1}{K_{+}(k)}\right\}-e^{i k d} R_{1}(s)\right] \\
& +i \mu C_{1} K_{+}(s) T(s), \\
-\Psi_{-}= & i \mu K_{+}(-s)\left[-e^{-i k d} \frac{1}{(s-k) K_{+}(-s)}-e^{i k d} R_{2}(s)\right] \\
& +i \mu C_{2} K_{+}(-s) T(-s)
\end{aligned}
$$

where $C_{1}$ and $C_{2}$ are as in Noble (1958). For large $k d$,

$$
\begin{aligned}
& C_{1} \sim \frac{e^{i k d}}{2 k}\left(1+\frac{i K_{+}(k)}{\mu(2 \pi)^{1 / 2}} d^{-1 / 2}\right), \\
& C_{2} \sim e^{-i k d} \frac{K_{+}^{\prime}(k)}{K_{+}(k)} .
\end{aligned}
$$

Now the quantity of interest is $D_{a}$, the transform of $f(x)$, which is proportional to the net compression force on the walls. From (5.2),

$$
D_{a}=-\frac{1}{K(s)}\left(e^{i d s} \Psi_{+}+F(s)+e^{-i d s} \Psi_{-}\right) .
$$

Using (5.9) the solution divides into two parts. There is a noninteraction term just the same as for a semi-infinitely long channel:

$$
f_{n i}(x)=\frac{-i \mu}{2 \pi K_{+}(k)} \int_{i a-\infty}^{i a+\infty} \frac{e^{i(s-k) d}}{(s-k) K_{-}(s)} e^{-i s x} d s .
$$


This is equivalent to (4.28), except that the $K(s)$ used is slightly different. There is also an interaction term,

$$
\begin{aligned}
f_{i}(x)=-\frac{i \mu}{2 \pi} \int_{i a-\infty}^{i a+\infty}\left\{e^{i k d} R_{1}(s)\right. & \frac{K_{+}(s)}{K_{-}(s)} e^{i d s}+e^{-i k d} R_{2}(s) e^{-i d s} \\
& \left.-C_{1} T(s) \frac{K_{+}(s)}{K_{-}(s)} e^{i d s}-C_{2} T(-s) e^{-i d s}\right\} e^{-i s x} d s .
\end{aligned}
$$

In the depths of the channel, where $d-x \gg 0$ and $d+x \gg 0$, a long calculation shows that

$$
f_{n i}(x) \sim \frac{e^{-i k d}}{i \sqrt{\pi}} \frac{k^{1 / 2}}{K_{+}(k)} \frac{e^{i k(d-x)}}{[k(d-x)]^{1 / 2}}+O\left(\{k(d-x)\}^{-1}\right)
$$

and

$$
f_{i}(x) \sim-\frac{1}{2 \sqrt{\pi}} e^{i k d} \frac{e^{i k(d+x)}}{[k(c+x)]^{1 / 2}}+O\left(\{k(d+x)\}^{-1}\right) .
$$

The field in the depths of the channel thus appears to be due to a source at each end. The major difference between this case and the case of oblique incidence is that the interaction term in the latter is $O\left((k d)^{-1 / 2}\right)$ smaller than here, where both sources appear to have strengths of similar magnitude. Note also that whereas varying $\mu$ changes $f_{n i}$ via the factor $K_{+}(k)$, it has no effect on the interaction term, to this order.

Acknowledgments. I should like to thank Professor F. G. Leppington for suggesting this problem, and for considerable assistance. The work was supported by the Science and Engineering Research Council.

Appendix. (a) Product factorisation of $K(s)=(\gamma a)^{-1} e^{-\gamma a} \sinh \gamma a$. The factor $(\gamma a)-\sinh \gamma a$ has no branch points, and so the infinite product representation theorem can be used. This gives

$$
L(s)=\frac{\sinh \gamma a}{\gamma a}=\frac{\sin k a}{k a} \prod_{m=1}^{\infty}\left(1-s^{2} / s_{m}^{2}\right)
$$

where $\left(s_{m}\right)$ is the set of roots of $(\gamma a)^{-1} \sinh \gamma a$, which lie at

$$
s_{m}=\left(k^{2}-\left(\frac{m \pi}{a}\right)^{2}\right)^{1 / 2} \quad(m=1,2, \ldots) .
$$

As $m \rightarrow \infty, s_{m} \sim \operatorname{im} \pi / a$, so we set

$$
L_{ \pm}(s)=\left(\frac{\sin k a}{k a}\right)^{1 / 2} \prod_{1}^{\infty}\left(1 \pm \frac{s}{s_{m}}\right) e^{ \pm i s a / m \pi} .
$$

We also need to factorise $e^{\gamma a}$, which we write as

$$
e^{\gamma a}=e^{a\left(f_{+}-f_{-}\right)} \text {. }
$$

The sum decomposition $\gamma=f_{+}+f_{-}$can be carried out using the general theory, giving

$$
f_{+}(s)=\frac{1}{\pi} \gamma \cos ^{-1}\left(\frac{s}{k}\right) \text {. }
$$


(b) Product factorisation of $K(s)=e^{-\gamma a}\left(1+\mu \gamma^{-1}\right)(\gamma \sinh \gamma a-\mu \cosh \gamma a)$. The factor $e^{-\gamma a}$ can be dealt with as in (A.4) and (A.5). For $1+\mu \gamma^{-1}$, write

$$
\begin{gathered}
\log M_{+}(s)+\log M_{-}(s)=\log \left(1+\mu \gamma^{-1}\right) \\
\frac{d}{d s} \log M_{+}(s)+\frac{d}{d s} \log M_{-}(s)=\frac{-\mu s}{\left(1+\mu \gamma^{-1}\right)\left(s^{2}-k^{2}\right)^{3 / 2}} .
\end{gathered}
$$

We can now divide this into partial fractions, and perform a sum decomposition as above to give

$$
\begin{aligned}
\frac{d}{d s} \log M_{+}(s)= & -\frac{1}{2(s+k)} \\
& -\frac{\mu}{2}\left\{\frac{f_{+}(s)-f_{+}(p)}{s-p}+\frac{f_{+}(s)-f_{+}(-p)}{s+p}\right\}
\end{aligned}
$$

where $p=\left(k^{2}+\mu^{2}\right)^{1 / 2}$. Fuller details are in Noble (1958), p. 89. The final factor is

$$
N(s)=\gamma \sinh \gamma a-\mu \cosh \gamma a \text {. }
$$

Suppose $\left(\alpha_{m}\right)$ is the set of roots of

$$
\pi \alpha_{m} \tan \pi \alpha_{m}=-\mu a
$$

then the roots of $N$ lie at $\left(s_{m}\right)$, where

$$
s_{m}=\left(k^{2}-\frac{\pi^{2} \alpha}{a^{2}} m^{2}\right)^{1 / 2} \quad(m=1,2, \ldots) .
$$

Since $N$ has no branch points, its infinite product representation is

$$
N(s)=(k \sin k a-\mu \cos k a) \prod_{1}^{\infty}\left(1-\frac{s^{2}}{s_{m}^{2}}\right) .
$$

Because $\alpha_{m} \sim m(m \rightarrow \infty)$, which is easily seen graphically, $s_{m} \sim \operatorname{im\pi } / a(m \rightarrow \infty)$. Therefore, we set

$$
N_{ \pm}(s)=(k \sin k a-\mu \cos k a)^{1 / 2} \prod_{1}^{\infty}\left(1 \pm \frac{s}{s_{m}}\right) e^{ \pm i s a / m \pi} .
$$

\section{REFERENCES}

Evans, D. V. \& Morris, C. A. N. (1972a), J. Inst. Maths. Applics., 9, 198-204

Evans, D. V. \& Morris, C. A. N. (1972b), J. Inst. Maths. Applics., 10, 1-9

French, M. J. (1977), J. Mech. Engng. Sci., 19, no. 2, 90-92

Havelock, T. N. (1929), Phil. Mag., 8, 569-576

Jones, D. S. (1952), Proc. Camb. Phil. Soc., 48, 118-134 
Jones, D. S. (1964), The theory of electromagnetism. Oxford, Pergamon Lamb, H. (1932), Hydrodynamics Cambridge University Press

Newman, J. N. (1974), J. Fluid Mech., 66, 97-106

Noble, B. (1958), Methods Based on the Wiener-Hopf Technique... London, Pergamon

Salter, S. H. (1974), Nature, 249, 5459

Thomas, J. R. (1981), Ph.D. Thesis, Bristol University

Ursell, F. (1947), Proc. Camb. Phil. Soc., 43, 374-382 